\title{
Postpartum Shock
}

National Cancer Institute

\section{Source}

National Cancer Institute. Postpartum Shock. NCI Thesaurus. Code C114404.

Severe loss of blood pressure leading to inadequate tissue perfusion after the delivery of the fetus. 